\title{
Polymeric nanomicelles: a potential hazard for the cardiovascular system?
}

\author{
"...current investigations on inorganic nanoparticles \\ have shed some light on the physiological, cellular and \\ molecular mechanisms for cardiovascular disorders."
}

\begin{abstract}
First draft submitted: 24 March 2017; Accepted for publication: 5 April 2017; Published online: 19 May 2017

Keywords: atherosclerosis $\bullet$ biomechanical forces $\bullet$ cardiovascular system $\bullet$ nanotoxicology - polymeric nanomicelles
\end{abstract}

The application of nanotechnology is rapidly increasing in both high-tech industries and biomedical fields, raising concerns over the potential health risk associated with exposure to nanomaterials. Since the urgency of nanomaterials toxicology was formally put forward in 2003 [1,2], many studies have discussed the biological effects of exposure to series of nanomaterials, including airborne ultrafine particles, titanium, zinc, silver, silica, iron and carbon-based lipid. In particular, these evidences based on epidemiologic, in vitro and in vivo studies suggested that a certain concentration of nanoparticles (NPs) are associated with increased risk of pulmonary complications, cardiovascular events and death from cardiovascular diseases. In addition to the shared nanostructures with other types of NPs, polymeric nanomicelles (PMs) have unique 'core-shell' structures and versatile functionalization design, and as such attracted massive attention in medical diagnostics, drug delivery, gene therapy, biomolecules detection and bioimaging [3]. The type of intermolecular forces drives block copolymer to format what is called PMs, which can be divided into several categories including hydrophobically assembled amphiphilic micelles, polyion-complex micelles, and micelles stemming from metal complexation. In contrast with inorganic NPs, current research on PMs mainly discusses its biological advantages while very few explore its potential complications to cardiovascular systems and the underlying mechanisms.

\section{Biocorona will be helpful for} understanding PM-cell interactions The NPs can be directly absorbed into local tissue site or into circulatory system for distribution via six principal routes including inhalation, intravenous, intraperitoneal, oral, dermal and subcutaneous, thereby leading to direct contact with the cardiovascular system. In absorption process, NPs are rapidly coated with macromolecules forming a socalled biocorona when they are introduced into a physiological environment [4]. Thus, the biocorona gives a biological identity to nanomaterials and alters the size, aggregation state, surface charge and interfacial properties that are distinct from its original synthetic identity. The physiological function of assorted proteins consist of this corona are generally involved in lipid transport, blood coagulation, complement activation, pathogen recognition or ion transport [5], and it is increasingly evident shown that corona formed on NPs play a key role in determining biological effect in vivo [6]. The structure and composition of corona depend on the synthetic identity of the nanomaterial, such as the chemistry, topography and curvature of the nanomaterial. Polymer nanomicelles possess various chemical composition, free residue and morphology (such as spheres, rods, vesicles, tubules and lamellae), which pro-

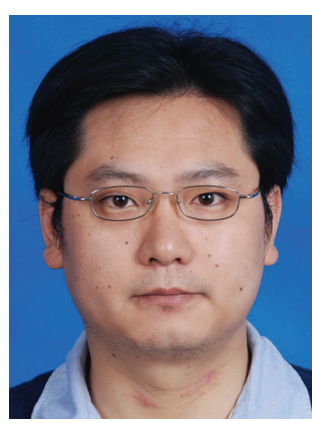

Yazhou Wang

Key Laboratory of Biorheological Science \& Technology, Ministry of Education,

State \& Local Joint Engineering Laboratory for Vascular Implants, College of Bioengineering, Chongqing University, Chongqing 400044, China

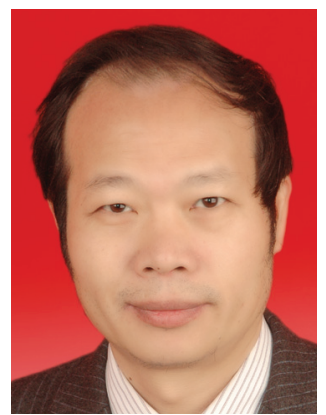

\section{Guixue Wang}

Author for correspondence:

Key Laboratory of Biorheological Science \& Technology, Ministry of Education,

State \& Local Joint Engineering Laboratory for Vascular Implants, College of Bioengineering, Chongqing University, Chongqing 400044, China wanggx@cqu.edu.cn 
vide PMs with more diverse synthetic identity. Thus, there is a reason to believe that PMs with corona will show completely different cell recognition or biological effects in vitro with in vivo [6]. Moreover, the corona formation may also lead to further dysfunction of the cardiovascular system through the mechanism which will be discussed as follows. Then, this may explain many existing inconsistencies between in vitro toxicity screening and in vivo studies, and make it necessary for re-evaluation of toxicity polymer nanomicelles, even if its polymer materials have good biocompatibility. For example, our recent study proved that the widely used PMs from the bioavailable copolymer, poly (ethylene glycol)-block-poly (e-caprolactone) (PEG-b-PCL), can impair the cardiovascular development in zebrafish under a certain polymer concentration situation [7].

\section{Possible mechanisms for cardiovascular dysfunction after exposure to PMs}

It is generally believed that NPs can cause oxidative stress, inflammation, apoptosis, autophagy and immune recognition, which induce cardiovascular cells injury and dysfunction. Despite the few evidences relating PMs to impairment of cardiovascular system, current investigations on inorganic NPs have shed some light on the physiological, cellular and molecular mechanisms for cardiovascular disorders.

\section{Oxidative stress \& inflammation}

Dysfunction of endothelial cells initiates the progression of atherosclerosis, which are closely associated with the development of cardiovascular diseases. Endothelial dysfunction can be triggered by oxidative stress, which then impact leukocyte adhesion and recruitment, platelet activation and thrombus formation. Meanwhile, inflammation is another common underlying mechanism for endothelial cells dysfunction. A large number of studies demonstrated that NPs adhering to membrane or uptake by cells (such as endothelial cell, macrophages and leukocytes) cannot only upregulate intracellular oxidative stress and reactive oxygen species (ROS), but also induce pro-inflammatory cytokines expression and release during absorption and transport in physiological environment [8].

"In contrast with inorganic NPs, current research on PMs mainly discusses its biological advantages while very few explore its potential complications to cardiovascular systems and the underlying mechanisms.'

During oxidative stress and inflammation, the circulating monocytes as precursors of macrophages are recruited to the activated endothelium, and migrate into the intima, where they engulf lipids and become foam cells, which further prolong inflammatory responses and promote atherosclerosis by accumulating lipids within destabilized plaques [9]. Currently, in vitro and in vivo evidences have demonstrated that NP exposure promotes lipid loading and induces foam cell formation in macrophages via different mechanisms including inflammatory responses, dysfunction of lysosomes, expression of receptors and inhibition of triglyceride hydrolysis [10]. Besides macrophages, many other types of cells, such as vascular smooth muscle cells (VSMCs), dendritic cells and mast cells, can also contribute to foam cells during inflammation [9]. Presently, there are no reports about NPs' exposure and foam cell formation in VSMC, which needs further studies.

Mast cells, as an essential cell of the innate immune system, are involved in mediating allergic reactions and inflammation via degranulation and the release of chemical mediators, which can promote atherosclerotic plaque growth and destabilization. There is evidence suggesting that mast cell activation may contribute to the adverse cardiovascular effects occurring after NPs' exposures [11]. NPs' exposures induce the releasing of complement components and inflammatory cytokines into the circulation which activate mast cells and mediate systemic inflammation and platelet activation. Noteworthy, either stimulation or inhibition of degranulation is related to physicochemical properties of NPs and types of NP-cell interactions (receptor recognition or endocytic internalization) [12]. However, the mechanism by which NP directly interacts with mast cells and influences degranulation is poorly understood and requires further examination.

\section{Autophagy}

Physiological autophagy benefits to a wide range of cellular events by clearing damaged organelles and proteins. However, autophagy has also been implicated in various diseases, including cancers and cardiovascular diseases. Autophagy can contribute to the inflammatory disorders, chronic ischemia, hypertension, repeated perfusion damage and heart failure [13]. Autophagy of VSMCs and endothelial cells can be triggered by inflammatory mediators, ROS, oxLDL, TNF- $\alpha$ and osteopontin. There is evidence indicating that NPs trigger the autophagy process in vascular endothelial cells [14], while it remains unknown whether PMs could regulate autophagy in cardiovascular cells.

\section{Apoptosis}

Apoptosis plays a critical role in the pathogenesis of various cardiovascular diseases and the inhibition of apoptosis is a promising strategy for therapeutic intervention of cardiovascular diseases. Macrophages' exposure [15] to NPs can result in the loss of the mito- 
chondrial membrane potential (MMP) and the accumulation of ROS, which activates the MAPKs and TGF- $\beta$ signaling pathways, leading to the activation of pro-apoptotic Bim and Bax as well as the cascade of caspases via mitochondria-related mechanisms, similar results were found in endothelial cells (ECs) [16].

\section{Harmful to cardiovascular development?}

Given the potential therapeutic benefits of nanomaterials in drug delivery and biomedical imaging, it would be inevitabe for a pregnant population to be routinely exposed to these products or dismissed from these therapies. Unfortunately, very limited research reports indicate its potential hazard on mother and fetus. Some evidences show that NP' exposure during pregnancy may have detrimental effects on maternal and fetal health at each stage of gestation [17]. However, an underlying mechanism is still unclear at this time associated with these outcomes including hostile gestational environment, gross abnormalities, impaired development and increased mortality.

Nanomaterials have been shown to translocate from the maternal system to fetal compartments through blood-embryo barrier, this makes fetal cardiovascular become an initial organ of exposure NPs. As a result, adverse effects of exposure NPs on fetal cardiovascular development should be paid immediate attention. Recently, we demonstrated that PEG-b-PCL nanomicelles can impair cardiovascular development in zebrafish. The reason is that these PMs inhibit zebrafish embryonic angiogenesis by upregulating p 53 expression and inducing apoptosis [7]. In general, bFGF, VEGF, Dll4/Notch, Slit3-Robo4, Hippo - YAP, NRG-1/ ErbBs, Angl/Tie2 and Wnt signaling pathway involve in the regulation of neovascularization, cardiac system venous return and pericardium development, and heart development, which may provide some underlying mechanism associated with cardiovascular system development damage induced by PMs' exposure.

\section{Biomechanical forces, a distinct perspective?}

The initiation and progression of atherosclerosis is influenced by biomechanical forces, with plaques developing predominantly near to side branches and/or bends in arteries. Blood flow at these sites is disturbed due to the uneven luminal geometry, leading to disturbed flow, for example low shear stress and excessive high shear stress [18]. Research from our lab and others suggest that low local endothelial shear stress (ESS) leads to atherosclerosis, and excessive high ESS is associated with plaque ruptures or intimal ulcerations. It is possible that disturbed flow also may influence the process of interaction between PMs with vascular cells when PMs are circulating in bloodstream. Thus, the dynamic assessment of exposure PMs appears very important under more physiological conditions. For example, biocorona of NPs shows different structure and composition between static-flow and dynamic-flow environments [19]. This possibly affects PMs-vascular wall interactions, such as recognition, adhesion, endocytosis, transport and deposition, and may in turn influence local blood flow.

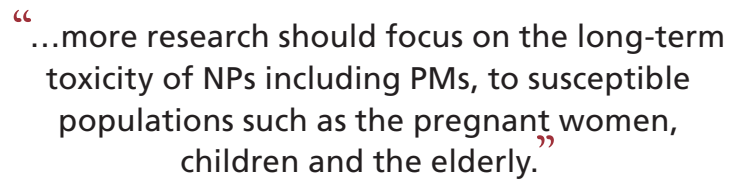

Furthermore, plaque structural stress (PSS) is determined partially by plaque composition/morphology and the material properties of tissues. As more polymer nanomicelles have been recently proposed for atherosclerosis detection and treatment via targeting the intimal macrophages, foam cells and endothelial cells [20], one would expect that internalization of PMs into atherosclerotic plaques could possibly alter either plaque architecture or composition, thereby increase or decrease in PSS. However increased PSS levels may be a principal mechanism through which plaques rupture, resulting in thrombosis and sudden ischemic clinical events. Although there is no direct evidence demonstrating a correlation between cardiovascular exposure to nanomaterials with alteration of ESS or PSS, it could serve as a distinct perspective for understanding cardiovascular damage after PMs' exposure.

\section{Looking onward}

Although it is known that some NPs exert nanotoxicity in some settings, few or no studies focus on the potential hazards on cardiovascular system especially after polymer nanomicelles exposure. Understanding its underlying mechanisms will contribute to the design of safe polymer nanomicelles for potential applications in drug delivery and bioimaging.

First, the underlying mechanisms for the potential hazards after PMs' exposure are unknown. Given the increasing knowledge on the cardiovascular toxicity of inorganic or metal NPs, it will be valuable to examine the oxidative stress, inflammation, apoptosis, autophagy and immune recognition upon PMs' exposure in different cell types or in vivo.

Second, further understanding the correlation between NP-cell interaction, particularly biocorona in physiological environment, may help the better modification of PMs for clinical uses. It is thus necessary to reassess PMs, even with good biocompatibility polymer from block materials.

Furthermore, while many studies focus on the acute systemic effects of nanomaterials, more research should focus on the long-term toxicity of NPs includ- 
ing PMs, to susceptible populations such as the pregnant women, children and the elderly.

\section{Financial \& competing interests disclosure}

The authors gratefully acknowledge funding from the National Natural Science Foundation of China (31370949, 11332003), National Key Technology R\&D Program of China

\section{References}

1 Brumfiel G. Nanotechnology: a little knowledge. Nature 424(6946), 246-248 (2003).

2 Service RF. Nanomaterials show signs of toxicity. Science 300(5617), 243 (2003).

3 Zhang Y, Huang Y, Li S. Polymeric micelles: nanocarriers for cancer-targeted drug delivery. AAPS PharmSciTech 15(4), 862-871 (2014).

4 Monopoli MP, Aberg C, Salvati A, Dawson KA. Biomolecular coronas provide the biological identity of nanosized materials. Nat. Nanotechnol. 7(12), 779-786 (2012).

5 Walkey CD, Chan WC. Understanding and controlling the interaction of nanomaterials with proteins in a physiological environment. Chem. Soc. Rev. 41(7), 2780-2799 (2012).

6 Ritz S, Schottler S, Kotman N et al. Protein corona of nanoparticles: distinct proteins regulate the cellular uptake. Biomacromolecules 16(4), 1311-1321 (2015).

7 Zhou T, Dong Q, Shen Y et al. PEG-b-PCL polymeric nano-micelle inhibits vascular angiogenesis by activating p53-dependent apoptosis in zebrafish. Int. J. Nanomedicine 11, 6517-6531 (2016).

8 Moller P, Christophersen DV, Jacobsen NR et al. Atherosclerosis and vasomotor dysfunction in arteries of animals after exposure to combustion-derived particulate matter or nanomaterials. Crit. Rev. Toxicol. 46(5), 437-476 (2016).

9 Bennett MR, Sinha S, Owens GK. Vascular smooth muscle cells in atherosclerosis. Circ. Res. 118(4), 692-702 (2016).

10 Cao Y, Long J, Ji Y et al. Foam cell formation by particulate matter (PM) exposure: a review. Inhal. Toxicol. 28(13), 583-590 (2016).

11 Shannahan JH, Kodavanti UP, Brown JM. Manufactured and airborne nanoparticle cardiopulmonary interactions: a review of mechanisms and the possible contribution of mast cells. Inhal. Toxicol. 24(5), 320-339 (2012).
(2016YFC1102305, 2016YFC1101101). The authors have no other relevant affiliations or financial involvement with any organization or entity with a financial interest in or financial conflict with the subject matter or materials discussed in the manuscript apart from those disclosed.

No writing assistance was utilized in the production of this manuscript.

12 Alsaleh NB, Persaud I, Brown JM. Silver nanoparticledirected mast cell degranulation is mediated through calcium and PI3K signaling independent of the high affinity IgE receptor. PLoS ONE 11(12), e0167366 (2016).

13 Lavandero S, Chiong M, Rothermel BA, Hill JA. Autophagy in cardiovascular biology. J. Clin. Invest. 125(1), 55-64 (2015).

14 Guo C, Yang M, Jing L et al. Amorphous silica nanoparticles trigger vascular endothelial cell injury through apoptosis and autophagy via reactive oxygen species-mediated MAPK/Bcl2 and PI3K/Akt/mTOR signaling. Int. J. Nanomedicine 11, 5257-5276 (2016).

15 Li Y, Liu Y, Fu Y et al. The triggering of apoptosis in macrophages by pristine graphene through the MAPK and TGF-beta signaling pathways. Biomaterials 33(2), 402-411 (2012).

16 Duan J, Yu Y, Li Y, Yu Y, Sun Z. Cardiovascular toxicity evaluation of silica nanoparticles in endothelial cells and zebrafish model. Biomaterials 34(23), 5853-5862 (2013).

17 Stapleton PA. Gestational nanomaterial exposures: microvascular implications during pregnancy, fetal development and adulthood. J. Physiol. 594(8), 2161-2173 (2016).

18 Brown AJ, Teng Z, Evans PC, Gillard JH, Samady H, Bennett MR. Role of biomechanical forces in the natural history of coronary atherosclerosis. Nat. Rev. Cardiol. 13(4), 210-220 (2016).

19 Hadjidemetriou M, Al-Ahmady Z, Kostarelos K. Timeevolution of in vivo protein corona onto blood-circulating PEGylated liposomal doxorubicin (DOXIL) nanoparticles. Nanoscale 8(13), 6948-6957 (2016).

20 Zhang J, Zu Y, Dhanasekara CS et al. Detection and treatment of atherosclerosis using nanoparticles. Wiley Interdiscip. Rev. Nanomed. Nanobiotechnol. 9(1), doi:10.1002/ wnan.1412 (2017) (Epub ahead of print). 